\title{
The effect of line configuration on perceived numerosity of dotted lines
}

\author{
ARADHNA KRISHNA \\ Columbia University, New York, New York \\ and \\ PRIYA RAGHUBIR \\ University of California, Berkeley, California
}

\begin{abstract}
Estimates of the number of objects in a line are made in many different situations. This paper demonstrates that besides the actual number of dots, aspects of line configuration affect the perceived numerosity of dotted lines. Experiment 1 provides evidence that the highly studied "clutter effect" in distance perception research replicates to the numerosity domain so that lines made up of more segments are perceived to contain more dots. Experiments 2-5 provide nomological validity for the recently proposed "direct distance" effect in distance perceptions by showing that numerosity perceptions are higher the greater the euclidean length between the line end points and by manipulating euclidean length in three orthogonal ways: the relative length of segments (Experiment 2), the angle between segments (Experiment 3), and the general direction of segments (Experiment 4). Experiment 5 conceptually replicates the results of Experiments 2-4 utilizing stimuli-based versus memory-based judgments and a discrimination task. Experiments 6 and 7 extend the research on spatial perception by demonstrating that the use of euclidean length as a source of information is inversely related to line width, with width varied through clutter (Experiment 6) and total line length (Experiment 7). Overall, the results demonstrate that the robustness of the euclidean length effect is contingent on the salience of alternative spatial heuristics-specifically, euclidean width. Theoretical implications are discussed.
\end{abstract}

Estimates of the number of objects in a line are made in many different situations. For example, for determining how long a traffic jam will take to clear, radio traffic reports use helicopters to estimate the number of cars on the freeway. Estimates of enemy strength (e.g., number of tanks, number of soldiers) are made through aerial satellite pictures. Estimates of migrating geese are made by looking at the formation of geese in the sky and making a judgment. It has also been demonstrated that estimating number, as opposed to counting or enumerating, leads to biases in numerosity judgments (Smitsman, 1982; van Oeffelen \& Vos, 1982, 1984). In this paper we examine the effect of line configuration on perceptions of numerosity of dotted lines.

The paper extends the research on the effect of visual cues on distance perceptions (e.g., Allen \& Kirasic, 1985; Antes, McBride, \& Collins, 1988) and numerosity perceptions (Aoki, 1977; Dixon, 1978). Although perceptions

We wish to thank the participants at the Columbia-New York University-Yale Marketing Colloquium at Columbia University (May 1993) for their comments and suggestions. The comments of Imran Currim, Gita Venkataramani Johar, Avijit Ghosh, Don Lehmann, Geeta Menon, Michel Pham, Bernd Schmitt, Nader Tavasolli, and Yehoshua Tsal on an earlier draft were very useful. We also wish to thank David Wooten for his assistance in data collection. A.K. is affiliated with the Graduate School of Business at Columbia; P.R., with the Haas School of Business at Berkeley. Correspondence should be addressed to A. Krishna, 511 Uris Hall, Columbia University, New York, NY 10027 (email: akj1@columbia.edu). of lengths of lines have been studied previously, there is little research on numerical perceptions of dotted lines (for exceptions, see Dixon, 1978, and Gelman, 1969) and no previous research on numerical perceptions of nonstraight dotted lines. We extend the research on numerical perceptions by focusing on nonstraight dotted lines. We augment the research on spatial perceptions (distance and numerical perceptions) by proposing additional spatial features that may affect perceptions of lines and demonstrating moderating factors and boundary conditions for effects previously demonstrated in the distance domain. Since there is little prior research on dotted lines, we first tested whether effects found in the distance domain replicate to the numerosity domain. Next, we tested for new spatial effects.

One effect that has been studied a lot in distance perception research is the "clutter effect," which proposes that the greater the clutter in a line caused, for example, by intersections, barriers, turns - the greater in length the line will be perceived to be (Sadalla \& Magel, 1980; Sadalla \& Staplin, 1980). Another effect that has been documented in a distance perception context is the euclidean length effect, which proposes that the greater the euclidean length between end points of a nonstraight line, the greater the estimates of length of the line (Raghubir \& Krishna, 1996). Raghubir and Krishna proposed that euclidean length is used as a heuristic in estimating line length because of its salience and that its use is partially automatic in nature. 
In Experiment 1 we first show that the clutter effect observed for distance perceptions also holds for perception of number of dots along a line. We then explore the euclidean length hypothesis. We separately vary three factors that affect the euclidean length of a line: the relative length of segments (Experiment 2), the angle between segments (Experiment 3 ), and the overall general direction of segments (Experiment 4). The latter two have also been studied for distance perceptions with similar results (see Raghubir \& Krishna, 1996). Experiment 5 conceptually replicates the results of Experiments 2-4 utilizing stimuli-based versus memory-based estimates.

Experiments 6 and 7 extend the spatial perception literature by proposing that another spatial feature, namely the euclidean width of the line (where euclidean width is defined as the widest part of the straight line connecting the end points of the line configuration) affects the use of euclidean length as a source of information in estimating number. The larger the euclidean width, the greater is its salience relative to that of euclidean length. Thus, euclidean width moderates the euclidean length effect. Euclidean width of the line is varied through clutter (Experiment 6 ) and total line length (Experiment 7). Experiments 6 and 7 also test for the direct effect of clutter and exposure time (Experiment 6 ), as well as total line length and number of dots (Experiment 7), on perceived numerosity. Results show that increasing the number of dots increases perceived numerosity, but is subject to a regression to the mean effect. Further, although exposure time does not moderate the euclidean length effect, longer exposure times are associated with higher numerosity estimates. We also found that the clutter effect is moderated by other variables. In addition, we obtained mixed findings for the effect of total line length. Finally, while testing for the size of effects, we found that euclidean length is as good if not better predictor of estimated numerosity as the actual number of dots in a configuration.

\section{LITERATURE REVIEW}

The effect of visual cues on perceived numerosity has been studied for a long time (see Oppel, 1855; cited in Thorndyke, 1981). In addition, the literature on distance perceptions in the fields of cognitive psychology (e.g., Allen \& Kirasic, 1985; McNamara, 1986; McNamara, Ratcliff, \& McKoon, 1984), environmental psychology (e.g., Sadalla \& Staplin, 1980), and urban planning (e.g., Antes et al., 1988) has implications for perceived numerosity. We draw on both the literature on numerosity perception and the literature on distance perception to understand how people estimate the number of objects (e.g., dots) in a line.

\section{Numerosity Perceptions}

The numerosity literature documents that when subjects quantify a set of dots, they do not always count by enumeration (i.e., one at a time) and that the arrangement of dots has an important effect on the speed and accuracy of counting (Aoki, 1977; Atkinson, Cambell, \& Francis, 1976a, 1976b; Beckwith \& Restle, 1966; Freeman, 1912; van Oeffelen \& Vos, 1982, 1984). Wolters, van Kempen, and Wijlhuizen (1987) have provided evidence that quantification of a small number of objects without counting may be done by pattern recognition rather than by subitizing (i.e., immediate apprehension of number).

Pattern recognition may lead to biases in number perception. Frith and Frith (1972) demonstrated the "solitaire illusion," whereby one large cluster appeared to contain more elements than did several small clusters. Ginsburg (1978) showed that regular sets of dots were overestimated whereas random sets were underestimated. Van Oeffelen and Vos $(1982,1984)$ have attempted to account for the number-pattern interaction on the basis of quantitative theory. Their approach is to develop an algorithm to simulate how a human perceiver deciphers the patterning in question.

The numerosity literature discussed in the previous paragraph focuses on 2-D dot patterns and specifically excludes lines of dots (van Oeffelen \& Vos, 1982), on which we focus in this paper. The exceptions are research by Dixon (1978) and Gelman (1969). Dixon explored numerosity perceptions of uniformly spaced rows of dots and found that incongruity between length and numerosity of an array increases the time required to estimate number. This result is consistent with Gelman's research showing that children use the length of the line in estimating the number of dots in a straight line.

\section{Distance Perceptions}

The literature on biases in distance perceptions is relevant for studying biases in numerosity perceptions, as people may use similar processes for estimating distance and number (Morris \& Rule, 1988). In a study on assimilation and contrast effects in sequential judgments, Morris and Rule found that judgments of magnitude, whether elicited using length estimates or numerosity estimates, were subject to similar biases and were highly correlated. Similarly, Luccio and Rodani (1983) found evidence demonstrating that numerosity and distance judgments have common aspects.

An area that has received much attention from distance perception researchers is the clutter effect. Clutter pertains to the presence of additional spatial features that need to be visually processed, and researchers have shown that the presence of landmarks (Allen, 1981; Allen, Siegel, \& Rosinski, 1978), barriers (Kosslyn, Pick, \& Fariello, 1974; Nasar, 1985; Newcombe \& Liben, 1982), intervening points (Thorndyke, 1981), and intersections (Sadalla \& Staplin, 1980) increases perceived distance. Thorndyke found this effect using two points on a map separated by zero to three (cluttering) cities. Kosslyn et al. found this effect using two dots in a room separated by (cluttering) barriers. Sadalla and Staplin found it using one, four, or seven intersections along a path both in a laboratory and in a field setting.

Researchers have also tried to replicate the clutter effect using "turns" but have had mixed findings. Whereas Lee (1973) and Sadalla and Magel (1980) found evidence 
for overestimation of length as a function of the number of turns in the lines, Herman, Norton, and Klein (1986) found that paths with $2 / 4 / 8$ turns were perceived to be no longer than equidistant paths with $0 / 1 / 2$ turns, respectively. Raghubir and Krishna (1996) recently proposed that the mixed findings may be due to a second effect associated with turns, namely the euclidean length between the end points of the line. They demonstrated that the shorter the euclidean length, the shorter the perceived length of the line. The fact that turns increase clutter but decrease euclidean length can result in mixed findings regarding the effect of turns on perceived numerosity. Raghubir and Krishna proposed that euclidean length acts as a heuristic that subjects use to make an initial estimate of length that they later adjust in piecemeal, systematic processing of the line.

Although the clutter and euclidean length effects have been studied in the distance domain, they have not been studied in the numerosity domain. In addition, they have not been systematically varied together. Further, although the euclidean length effect has been studied by Raghubir and Krishna (1996), further research is needed to determine how robust the effect is and under what conditions it holds. In this paper, after demonstrating that the euclidean effect moderates the clutter effect (Experiment 1), we manipulate euclidean length in three orthogonal ways and provide nomological validity for the euclidean length effect (Experiments 2-6). Further, we identify another spatial factor moderating the effect of euclidean length in a spatial perception task, namely the euclidean width of the configuration (Experiments 6 and 7). These experiments are now described.

\section{EXPERIMENT 1 \\ The Interactive Effects of Euclidean Length and Clutter}

Turns along a dotted line are a spatial feature that add clutter to the dotted line and also reduce the euclidean length between the end points of a line. On the other hand, intersections and spaces within a line increase clutter and also increase the euclidean length between the end points of a line. On the basis of the clutter effect, we predicted that lines with features such as turns, intersections, and gaps would be perceived as containing more dots than a control line without these features. However, on the basis of the euclidean length hypothesis, we predicted that a line with an intersection or gaps would be estimated as longer than a line with turns.

\section{Method}

Subjects were 59 graduate students of business at a large northeastern university who completed the experimental task during a regularly scheduled class. We used a 4 (spatial stimuli: intersection, gaps, turns, and control) $\times 2$ (orientation: horizontal or vertical) mixed design. The spatial stimuli factor was administered within subjects and the orientation factor was administered between subjects. The control line was a straight, uncluttered line. The orienta- tion (vertical or horizontal) of the stimuli was counterbalanced as this has been shown to affect estimates of length (Müller-Lyer illusion, Brosvick \& Cohen, 1988; Finger \& Spelt, 1947). The horizontal orientation is presented in Figure 1A. In the vertical orientation the map was rotated $90^{\circ}$. Because of potential biases that unequal spacing could cause (Dixon, 1978), dots were equally spaced within and across manipulations, except when the manipulation itself called for a space between dots.

Each subject was shown four lines (Figure 1A). Each line contained 19 dots. Subjects were then instructed to turn the page (after seeing Figure 1A for as long as they wished) and to estimate the number of dots in each line. At the beginning of the exercise, subjects were instructed not to turn to a previous or later page. They were told specifically not to count the number of dots. Each line was named by a randomly selected letter. After answering questions on demographics (including gender), and right- or left-handedness (as this has been shown to affect spatial judgments, e.g., Masin \& Agostini, 1991), subjects were debriefed and excused. The procedure took approximately $10 \mathrm{~min}$.

\section{Results and Discussion}

We found that subjects took between $20 \mathrm{sec}$ and $2 \mathrm{~min}$ to look at the stimuli. Neither gender nor handedness had an effect and these variables are not included in the analysis. Lines in the vertical orientation were perceived to have more dots than those in the horizontal orientation. However, this was significantly different only for the line with gaps $(p<.05)$ and not for lines with turns or intersections $(p>.2){ }^{1}$ The remainder of the analysis includes orientation as a design factor.

Overall, the results support the hypothesis that clutter increases estimated number, but the magnitude of this effect is reduced if clutter decreases the euclidean length between the end points of the line (Figure 1B). A repeated measures analysis of variance (ANOVA) including orientation as a between-subjects factor showed an overall effect of configuration on estimates of number of dots $[F(3,171)=19.46, p<.001$; within-subjects standard error, $\left.S E_{\mathrm{ws}}=0.37\right] .{ }^{2}$ As seen in the pairwise contrasts reported in Figure 1B, the control line was estimated to have significantly fewer dots $(M=15.5, S D=4.0)$ than the three others [turns $M=16.5, S D=4.1 ; t(58)=1.85$, $p<.07$; intersections $M=19.2, S D=5.2, t(58)=6.64$, $p<.001$; gaps $M=18.0, S D=4.8 ; t(58)=4.28, p<$ $.001]$. Thus, clutter due to turns, intersections, or gaps did lead to number estimates larger than the control (the straight, uncluttered) line.

In support of the euclidean length hypothesis, the line with turns was estimated to have fewer dots than the line with gaps $[t(58)=3.00, p<.004]$, and was also estimated to have fewer dots than the line with intersections $[t(58)=$ $5.31, p<.001]$. Thus, spatial features that decreased euclidean length reduced the clutter effect, versus spatial features that increased the euclidean length.

To summarize, in Experiment 1, we found that clutter through the presence of intersections, gaps, and turns increased perceived numerosity of a line. Hence, we were able to successfully replicate the clutter effect found in distance estimates to numerosity estimates. Furthermore, we showed that the euclidean length effect reduces the effect 
A
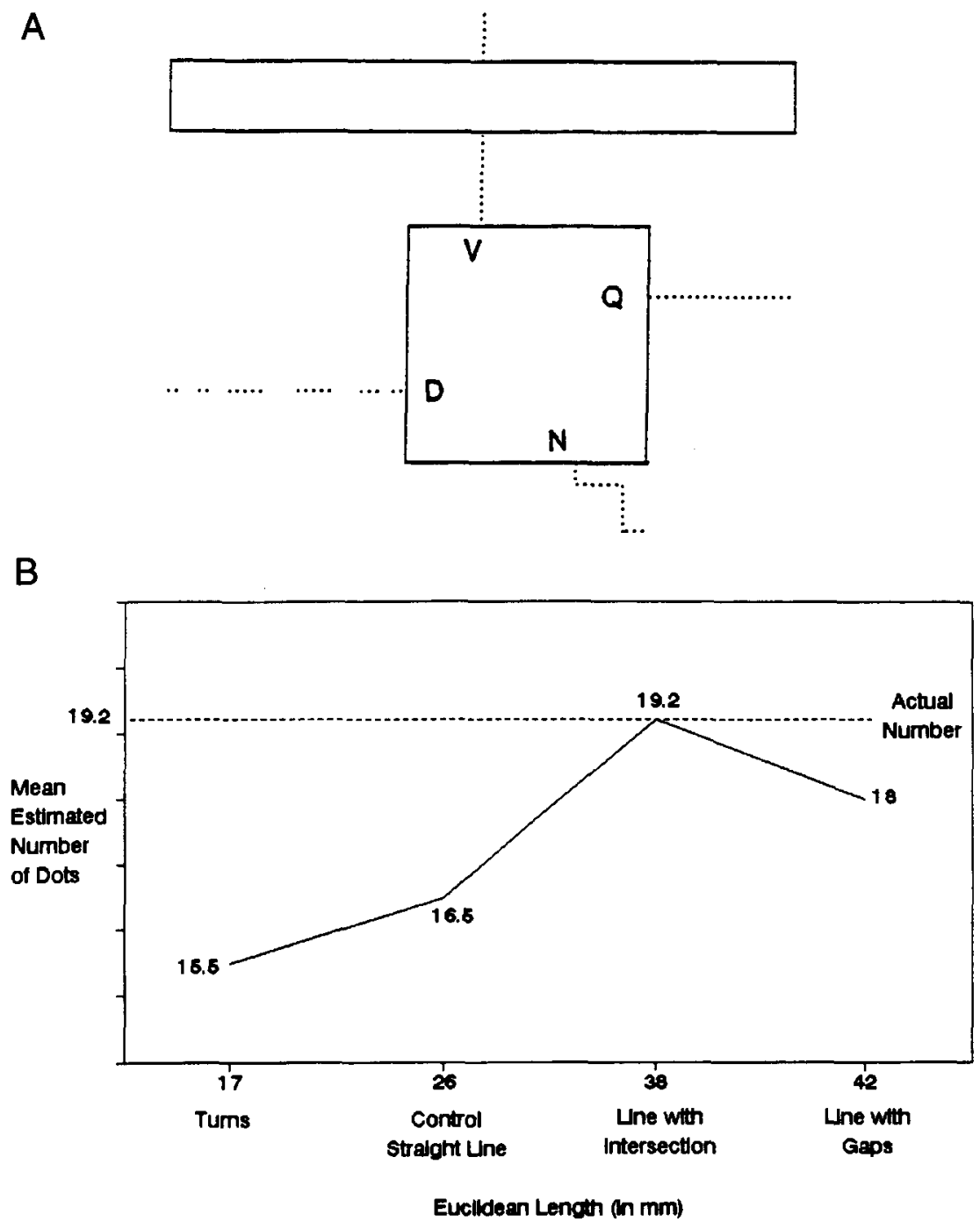

Figure 1. (A) Stimuli for Experiment 1 : straight line, turns, intersections, and gaps. (B) Experiment 1: straight line, turns, intersections, and gaps.

of clutter if clutter decreases the euclidean length of a line. We demonstrated that features that clutter and decrease euclidean length (such as turns) tend to decrease the perceived numerosity of a line and that features that clutter and increase euclidean length (such as intersections and gaps) tend to increase perceived numerosity, though all three lines with clutter were estimated to have more dots than the straight, uncluttered control line. This effect was found in spite of the fact that subjects could view the figure for as long as they wanted. Also, although subjects were instructed not to count dots, some subjects may have done so, and some may have counted in part and then extrapolated to form their perceptions. This leads us to have greater faith in our results.

In Experiments 2-4, we further investigated the effect of euclidean length, controlling for the presence and amount of clutter in the arrangement of dots on a line.

\section{EXPERIMENT 2 Length of the Line Segments}

One spatial feature that affects the euclidean length between the end points of a line made up of two segments is the relative length of these segments. When two segments are equal, the euclidean length between the end points is shorter than when they are of unequal lengths. For line segments making a $90^{\circ}$ angle, the hypotenuse is a direct measure of the euclidean length between the end points of the lines. Thus, in Figure $2 \mathrm{~A}$, line $\mathrm{W}$ is.composed of two equal segments, and its hypotenuse (euclidean length) is shorter than that of line F, which is composed of two unequally sized segments. This feature is orthogonal to other spatial features that bias distance perceptions such as the number of turns in the lines (Sadalla \& Magel, 1980, Experiment 1). In this experiment, 
A

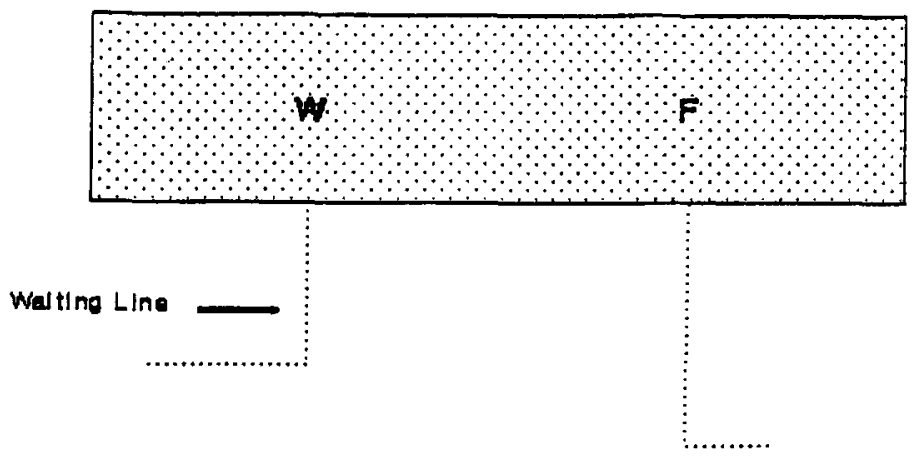

B

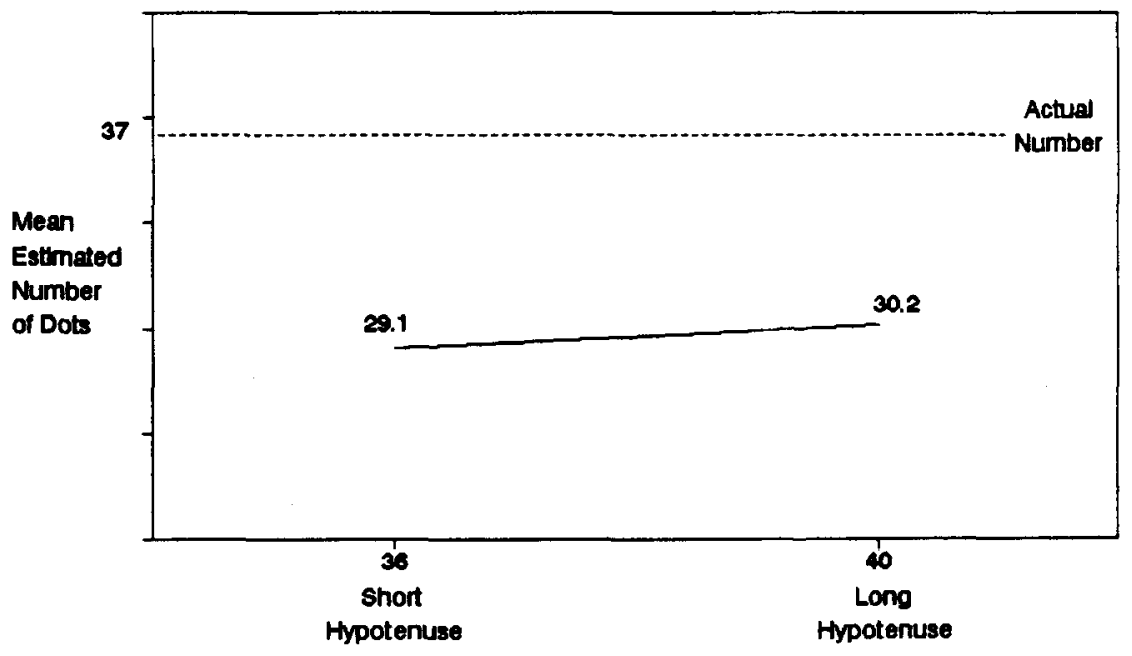

Euctideen Length (n $\mathrm{mm}$ )

Figure 2. (A) Stimuli for Experiment 2: long versus short hypotenuse. (B) Experiment 2: long versus short hypotenuse.

we wished to investigate whether the relative length of line segments affects numerosity perceptions. Since lines composed of two equal segments have a smaller euclidean length than do lines composed of two unequal segments, we expected that the former line would be perceived as having fewer dots than the latter.

\section{Method}

Fifty-five graduate students of business at a large northeastern university completed the experimental task during a regularly scheduled class. We used a within-subjects design in which subjects were presented a map (Figure $2 \mathrm{~A}$ ) that had a pair of lines with two segments connected with a $90^{\circ}$ angle. Both lines were of equal length, each composed of 37 dots. In one line the two segments had an equal number of dots ( 18 dots each with 1 dot connecting the two segments), whereas in the other line one segment ( 26 dots) was longer than the other ( 10 dots, 1 dot connecting the two segments). Dots were equally spaced within and across lines, so that the lines with segments of equal length were $25 \mathrm{~mm}$ each in length and had a hypotenuse (euclidean length) of $36 \mathrm{~mm}$, whereas the line with segments of unequal lengths had a hypotenuse of $40 \mathrm{~mm}$ between the longer segment $(38 \mathrm{~mm})$ and the shorter segment $(13 \mathrm{~mm})$. Randomly selected letters were used to name the lines in Experi- ments $2-4$. Subjects were asked to study the map closely and estimate the number of dots in each line.

In Experiments 2-4, the left-right orientation of each map was flipped for half the subjects. The potential effect of the verticalhorizontal orientation was controlled by having all the manipulations along the vertical axis. Left-right orientation of the stimuli did not have an effect in any of the three experiments and is not discussed further.

\section{Results and Discussion}

We expected that the line with the shorter hypotenuse (shorter euclidean length) would be estimated as having fewer dots than the line with the larger angle. The results supported this hypothesis (Figure 2B). The line with a smaller hypotenuse was estimated to contain marginally fewer dots $(M=29.1, S D=9.0)$ than the line with the longer hypotenuse $[M=30.2, S D=9.7 ; F(1,54)=2.39$, $p<.065$, one-sided, $S E_{\mathrm{WS}}=0.55$; see Figure 2B]. Both these estimates represent a significant underestimation of the actual number of dots $[t(54)=6.57$ and 5.17 , respectively, both $p s<.01]$.

The results of Experiment 2 are consistent with the euclidean length hypothesis that the shorter the euclidean 
length between two end points of a line, the lower the perceived numerosity of the line. However, there is a competing explanation for the results. In this experiment the two line segments were of unequal length. The $a b-$ solute length of segments can itself affect length perceptions, with longer lengths being underestimated relative to shorter ones (e.g., Dainoff, Miskie, Wilson, \& Crane, 1974). Therefore, the different absolute lengths of the segments, rather than the differential euclidean length, could be affecting our results. In the following experiment, we control for the length of segments and manipulate euclidean length through the size of the angle between segments.

\section{EXPERIMENT 3 Size of Angle}

The euclidean length of two equally long lines containing an equal number of turns is greater with a larger angle between line segments (see Figure 3A, where line $\mathrm{V}$ has a larger angle between its end points and a larger euclidean length than does line $\mathrm{D}$ ). This feature is orthogonal to other spatial features that bias distance perceptions, such as the number of turns in the lines ( $\mathrm{Sa}$ dalla \& Magel, 1980, Experiment 1), and controls for segment lengths that are equal across lines (Experiment 2). In this experiment, we wished to examine whether the size of the angle between segments affects numerosity perceptions in such a way that the wider the angle (and hence the larger the euclidean distance), the greater the perceived numerosity of a line of dots.

In a study related to urban planning, Byrne (1979) found that people's estimates of angles were distorted toward $90^{\circ}$. Ninety degrees, thus, seems to be a reference point for people. Thus in the present experiment, euclidean length was manipulated through size of the angle, controlling for the possible bias of distorted perceptions of angularity toward $90^{\circ}$.

\section{Method}

Fifty-five graduate students of business at a large northeastern university completed the experimental task during a regularly scheduled class. Subjects were presented a map (Figure 3A) that had a pair of lines with four segments each. The overall length was 25 dots for each line across four segments $(6,6,6$, and 7 dots). In one of the lines, the segments were connected to each other with a $45^{\circ}$ angle, whereas in the other they were connected with a $135^{\circ}$ angle. The acute angle and the obtuse angle diverge from a right angle by exactly $45^{\circ}$, thus controlling for the possible effect of angularity perception being distorted toward $90^{\circ}$. The euclidean length in the acute-angle line was $16 \mathrm{~mm}$ and that in the obtuseangle line was $34 \mathrm{~mm}$.

A

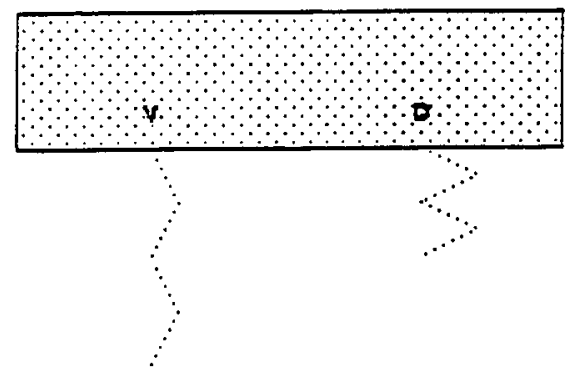

B

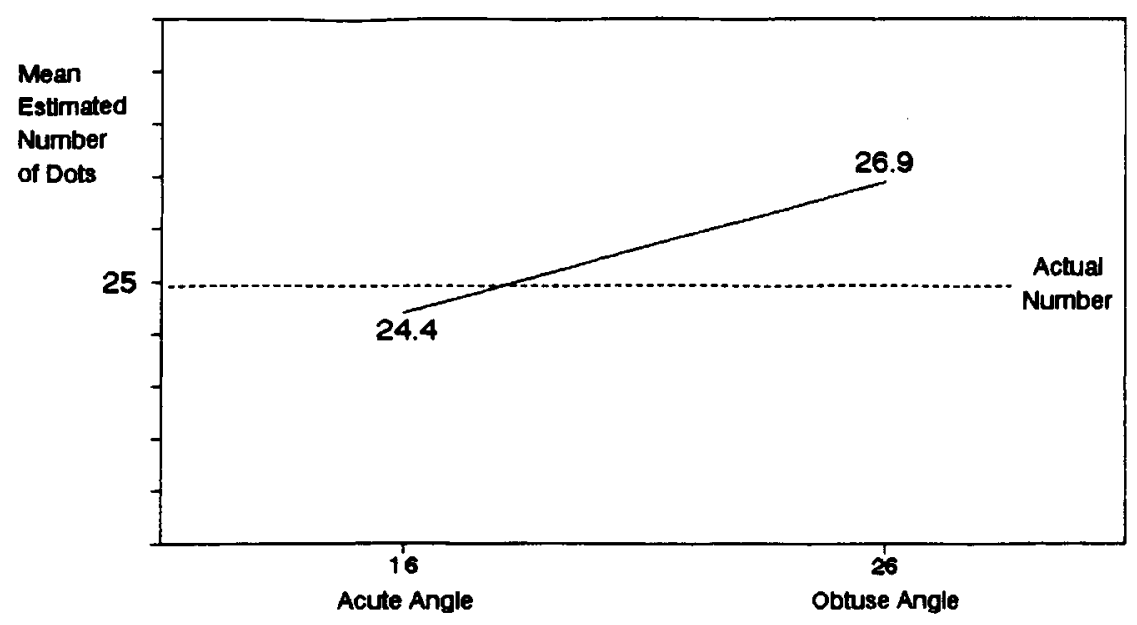

Euclldean Length (m mm)

Figure 3. (A) Stimuli for Experiment 3: acute versus obtuse angle. (B) Experiment 3: results. 


\section{Results and Discussion}

As expected, the line with the acute angle was estimated as having fewer dots $(M=24.4, S D=7.2)$ than the line with the obtuse angle $[M=27.0, S D=7.3, F(1,54)=$ $19.44, p<.01, S E_{\mathrm{ws}}=0.41$; see Figure 3B]. Therefore, the results support the hypothesis.

The results of Experiment 3 provide further evidence that the angle between segments of a line affects its perceived numerosity. One explanation for the results of Experiment 3 , however, may be that lines with larger angles (and not the euclidean length, per se) are associated with larger perceived number. In the next experiment we manipulated euclidean length, keeping the angle between segments the same, by manipulating the overall direction of segments.

\section{EXPERIMENT 4 Direction of Line Segments}

The euclidean length between the two end points of a multisegmented, nonstraight line is also affected by the general direction of the line segments. A line is unidirectional when the segments of the line follow the same general direction on both the east-west and north-south axes. Alternatively, a line in which segments retrace their direction on at least one of the two axes is a retraced line
(Figure 4A: Line $P$ is the retraced line and line $E$ is the unidirectional line). Simply stated, by retracing we mean turning back and going along the same direction that one just moved on, as opposed to continuing in the same direction. The line that retraces its direction has a shorter euclidean length between line end points than does the unidirectional line. The direction factor is orthogonal to other factors shown to affect numerosity perceptions, such as the presence of clutter (Experiment 1), relative length of segments (Experiment 2), and angularity of segments (Experiment 3).

\section{Method}

Fifty-five graduate students of business at a large northeastern university completed the experimental task during a regularly scheduled class. Subjects were presented a map (Figure 4A) that had a pair of lines with an identical number of dots (56) except that one of the lines was unidirectional, like a series of steps, whereas the other retraced its direction, like a snake. The euclidean length between the start and end of the retraced line was $22 \mathrm{~mm}$, whereas the euclidean length for the unidirectional line was $60 \mathrm{~mm}$. The two lines had an identical number of turns ( 9 each); the short segments had 4 dots and the long ones had 8 dots each. To control for angularity, we used right angles between all segments.

\section{Results and Discussion}

As expected, we found that the line that retraced its direction was estimated to contain fewer dots $(M=45.8$,

A
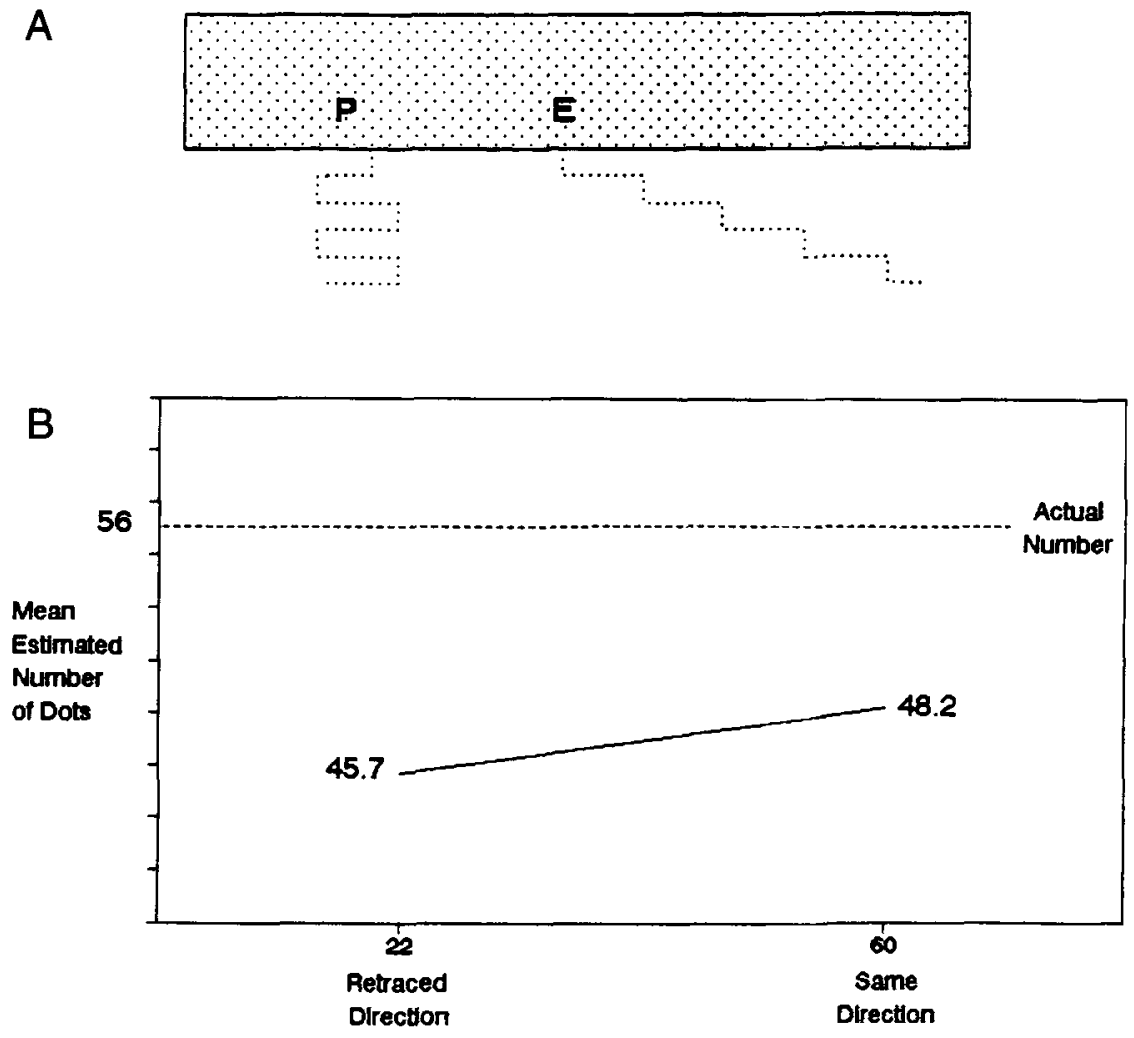

Euclldean Length (in mm)

Figure 4. (A) Stimuli for Experiment 4: same versus retraced direction. (B) Experiment 4: results. 
$S D=16.8)$ than the line that did not retrace its direction $\left[M=48.2, S D=15.1 ; F(1,54)=7.75, p<.007, S E_{\mathrm{WS}}=\right.$ 0.63 ; see Figure 4B].

The general direction of a line was found to affect the perceived numerosity of lines containing an identical number of dots. The line that retraced its direction was estimated to be shorter than the one that did not. This result, taken together with the results of Experiments 2 and 3 , provides convergent support for the euclidean length hypothesis. The relative length of segments, the angle between segments, and the overall direction of segments all affected the perceived numerosity of a line. A parsimonious explanation covering these factors is the euclidean effect.

\section{EXPERIMENT 5 Memory-Based Versus Stimuli-Based Judgments}

The procedure employed in Experiments 1-4 required subjects to view the stimuli and then turn the page to respond to the questions. Therefore, the responses may represent a response to memory-based stimuli. To the extent that memory-based representations are themselves biased, the results may reflect a memory-based distortion of spatial features. Therefore, it can be argued that in situations in which stimuli are physically present at the time of judgment (e.g., you are in front of two waiting lines and need to decide which to join), judgments would be less biased by the presence of spatial features. On the other hand, Raghubir and Krishna (1996, Experiments 4 and 5) showed that judgments may, in fact, be more inaccurate when they are stimuli-based due to sheer physical presence leading to the greater salience of the biasing euclidean length heuristic. To understand the extent to which our results were dependent on whether the experimental task is memory based or stimuli based, we conducted Experiment 5.

\section{Method}

Fifty-seven graduate students of business at a large northeastern university completed the experimental task during a regularly scheduled class. The procedure was similar to that of previous experiments, with the following differences: (1) Subjects were asked to provide their responses on the same page as the stimuli, rather than on the next page. (2) The dependent measure used was a task to estimate which line had fewer dots, rather than to give an exact estimate of the number of dots in each line. This was because with the stimuli directly in front of them, subjects would be more inclined to count if they were given an estimation task rather than the simpler discrimination task. (3) Subjects were exposed to three maps $^{3}$ (Figures 2A, 3A, and 4A), with order of presentation and left-right orientation counterbalanced. Subjects were asked to choose which of the two lines they believed had fewer dots.

\section{Results and Discussion}

Orientation and order of administration did not have main or interaction effects and are not reported. A binomial test was performed to test whether a significant majority perceived the line with a shorter euclidean length as having fewer dots. The results are presented in Figure 5 and conform to expectations.
Long versus short hypothenuse. The line with the equal segments was estimated to be shorter than the line with unequal segments by 41 out of 57 respondents $(z=$ $3.68, p<.01)$.

Acute versus obtuse angle. The line with the acute angles was estimated to be shorter than the line with the obtuse angles by 37 out of 57 respondents $(z=2.36, p<.01)$.

Same versus retraced direction. The line that retraced its direction was estimated to be shorter than the line that was unidirectional by 44 out of 57 respondents $(z=4.89, p<.01)$.

To summarize, the results of Experiments 2-4 were replicated using a discrimination task as opposed to an estimation task, with the stimuli directly present in front of the subjects. Therefore, even when the stimulus was directly in front of them, and they did not have to rely on a memory-based representation, subjects consistently demonstrated perceiving lines with shorter euclidean length as having fewer dots. It appears that people use the euclidean length between line end points to estimate number of dots in a nonstraight line.

However, in Experiments 1--5, lines differing in euclidean length were shown to subjects at the same time. This could make the difference in euclidean length more salient and increase its effect. On the other hand, it could be argued that with the two lines presented alongside it may be more apparent that the lines differ only in euclidean length (and not in number), and hence the euclidean length effect may be lower. In Experiments 6 and 7 we explored whether the euclidean effect is robust across experimental procedures. Not only did we show stimuli one at a time, but, unlike Experiments 1-5, in Experiments 6 and 7 we tested for the euclidean length effect under short, controlled exposure times.

More importantly, Experiments 2-5 show that euclidean length influences subjects' numerosity judgments when two lines of equal length, with an equal amount of clutter, and containing the same number of dots differ in euclidean length. Consistent with Raghubir and Krishna (1996), we believe this is due to the perceptual salience of euclidean length. Experiments 6 and 7 tested the generalizability of the euclidean length effect by systematically varying other spatial features that reduce the salience of euclidean length.

\section{EXPERIMENT 6 The Interactive Effects of Clutter and Euclidean Length}

We have proposed that people use the information provided by the euclidean length of a row of dots to estimate the number of dots in the line. However, given that we tested for this effect with nonstraight lines (lines with turns), there is another dimension of the line configuration that may affect perceived numerosity, namely the euclidean width of the configuration. In fact, in Experiments $2-5$, when the euclidean length of the configuration increased, the euclidean width decreased (see Figures $2 \mathrm{~A}, 3 \mathrm{~A}$, and $4 \mathrm{~A}$ ). 


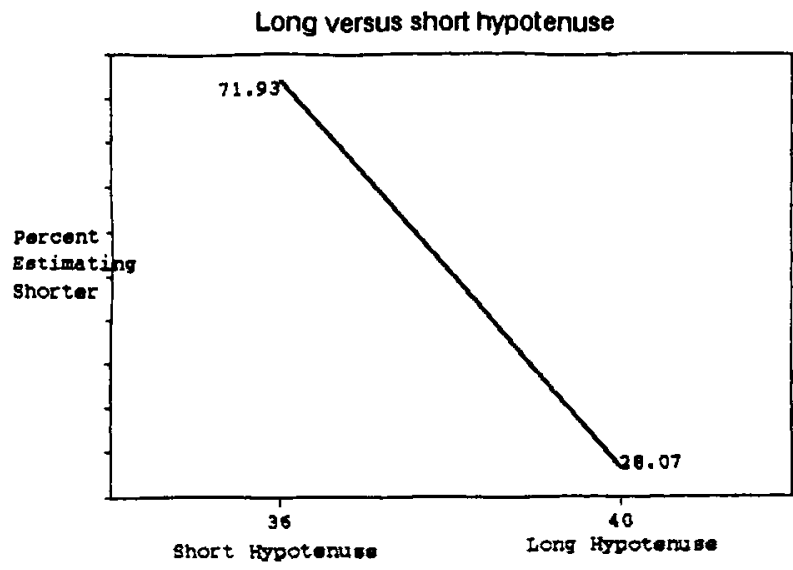

Euclidean Length (in min)

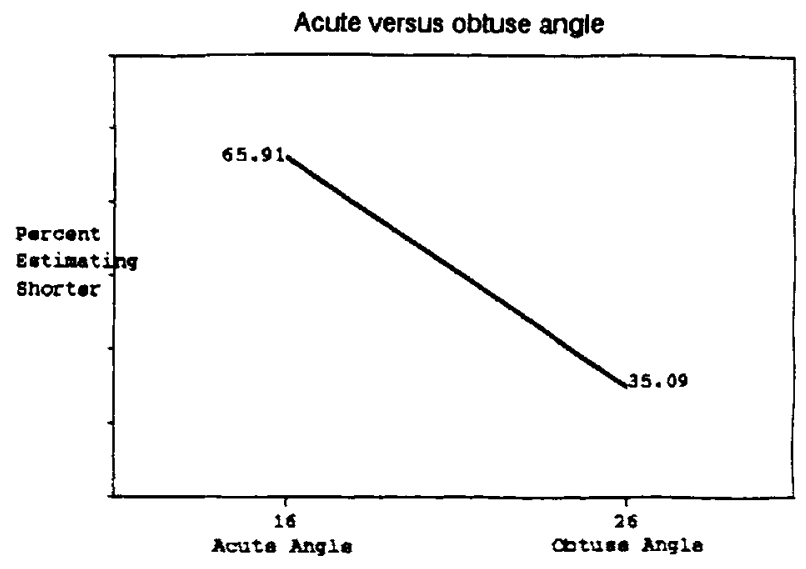

Duclidean Length (in )

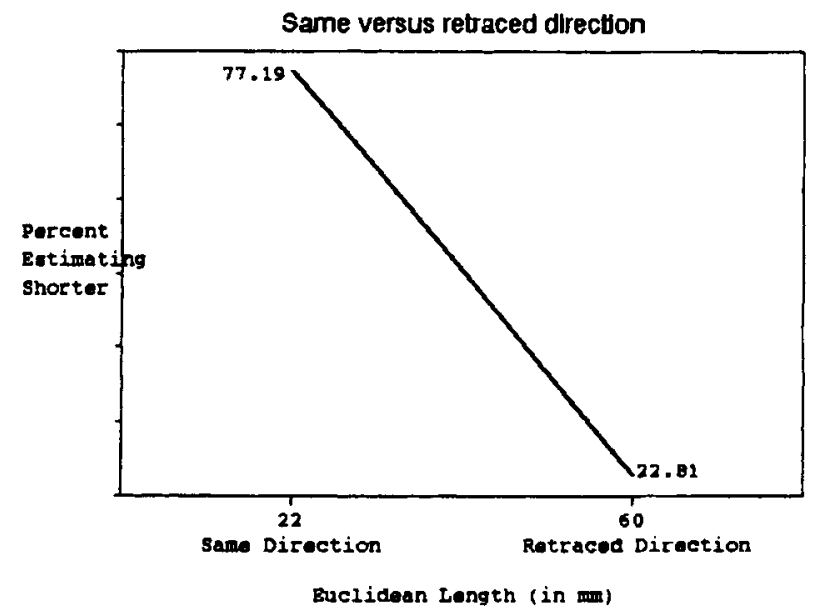

Figure 5. Experiment 5: results.

If angularity (i.e., the angle between line segments) is used to manipulate euclidean length, the euclidean width of the line configuration is a function of the number of line segments, or clutter (Figure 6A). The greater the number of line segments, for the same euclidean and total line length, the smaller the width of the overall dot configuration. We propose that the wider the euclidean width, the lower the salience of euclidean length. Hence, euclidean width should moderate the euclidean length effect in such a way that the lower the euclidean length/ width ratio, the lower the euclidean length effect. In other words, in an arrangement in which the predominant dimension is the length of the arrangement, euclidean length is more likely to be used as a source of information in estimating number and should exert a greater effect on the perceived numerosity than when both euclidean length and width are prominent.

The preceding paragraph shows that since clutter affects the euclidean width of the line, it may moderate the euclidean length effect. Thus far we have not tested for these interactive effects of euclidean length and clutter. In Experiment 1 we did not systematically vary both clutter and euclidean length. In Experiments 2-5 we held clutter constant and varied only euclidean length. Hence, we did not directly test whether euclidean length and clutter work interactively - that is, whether the strength of the euclidean length is moderated by the effect of clutter, and vice versa. In Experiment 6 we varied both clutter and euclidean length to test for the moderating effect of clutter on the euclidean length effect. We also controlled subjects' exposure time to single stimuli shown in multiple trials and varied it at two different levels.

\section{Method}

Subjects were 122 undergraduate students at a large business school who completed the experimental task during a regularly scheduled class for partial course credit. We used a $2 \times 2 \times 2$ (euclidean length $\times$ clutter $\times$ exposure time) mixed design, with euclidean length and clutter as within-subjects factors and exposure time to stimuli as a between-subjects factor. Euclidean length was varied at 50 and $100 \mathrm{~mm}$, and clutter at 5 segments and 10 segments. Exposure time to each stimuli was controlled at 10 or $15 \mathrm{sec}$. We call the two levels of each factor "low" and "high." The lines were similar to those in Experiment 3, in which we manipulated direct distance by manipulating the angle between segments using lines composed of more than two segments. All segments were of equal length (125 mm long) and contained 50 dots, ensuring equal dot spacing within and across stimuli (see Figure 6A). 
A

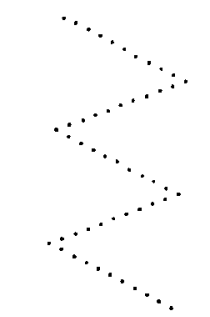

Low euclidean length low clutter

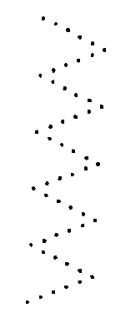

low cuclidean length high clutter

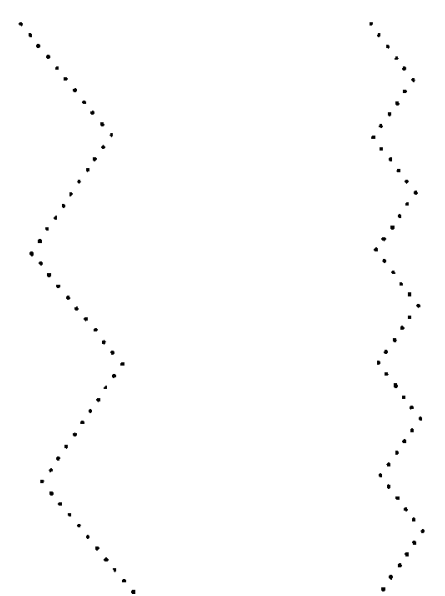

high euclidean length high euclidean length low clutter high clutter

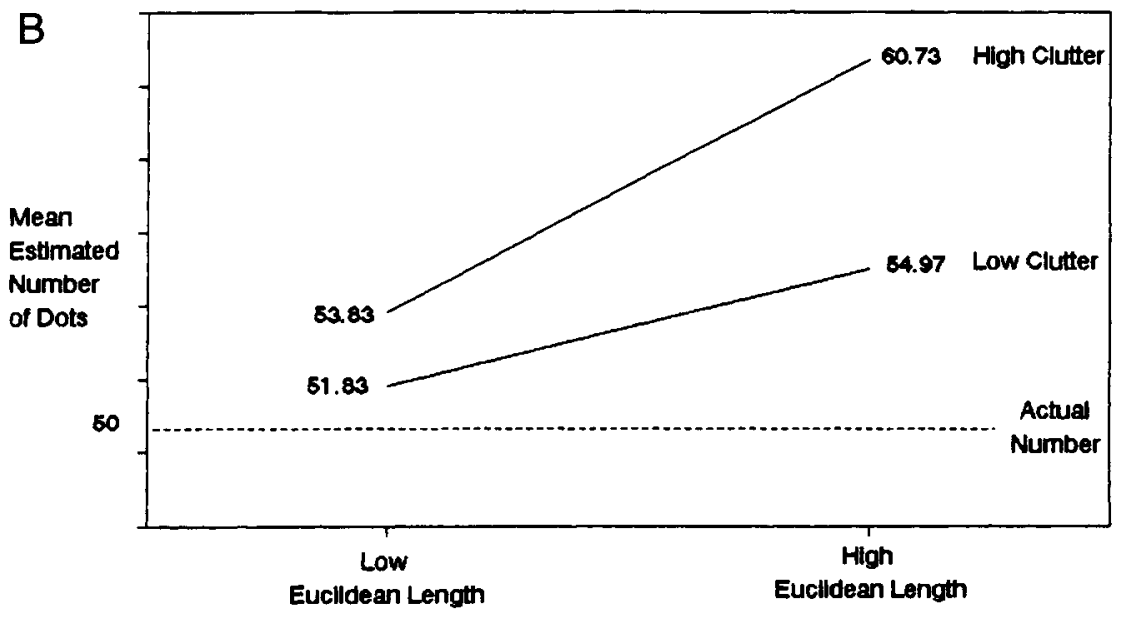

10 Seconds Exposure Time

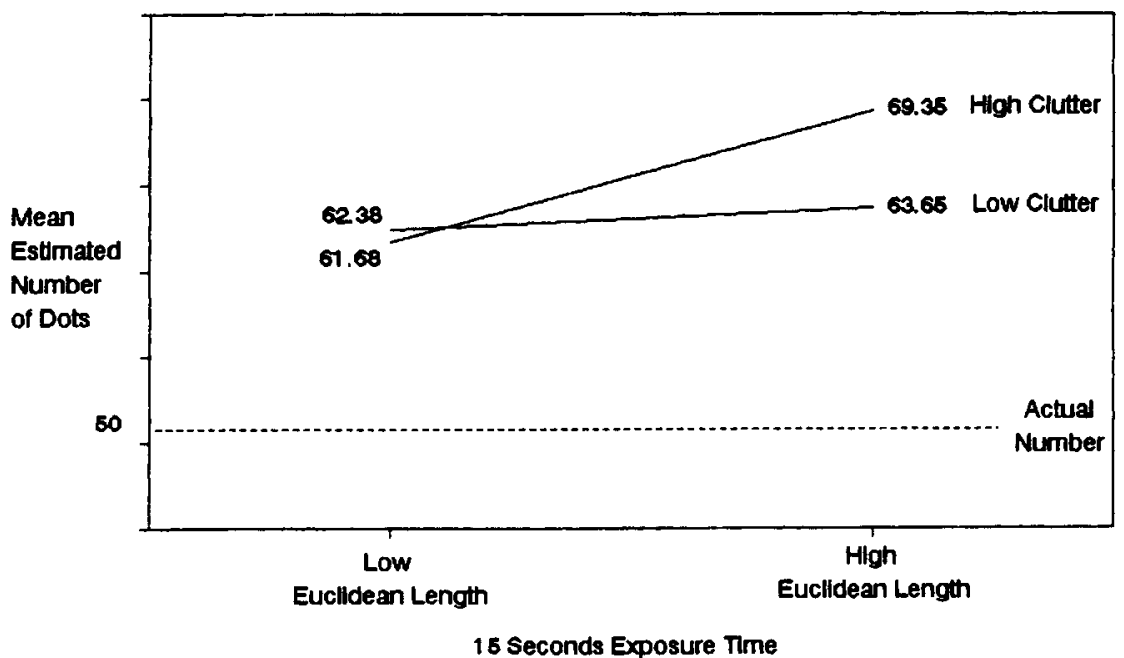

Figure 6. (A) Stimuli for Experiment 6: clutter, euclidean length, and exposure time. (B) Experiment 6: clutter, euclidean length, and exposure time. 
To control exposure time to the stimuli, subjects were shown the transparencies of the stimuli on an overhead projector. They were given a single sheet of paper to record their responses. They were asked to estimate the number of dots in each line and were told specifically not to count the number of dots. Finally, subjects were debriefed and excused. The procedure took approximately $15 \mathrm{~min}$.

\section{Results}

We did a repeated measures ANOVA with euclidean length and clutter as within-subjects factors, exposure time as a between-subjects factor, and estimate of number as the dependent variable.

Main effect of euclidean length. Consistent with Experiments $1-5$, the analysis revealed a significant main effect of euclidean length $[F(1,118)=14.77, p<.001$, $\left.S E_{\mathrm{ws}}=1.24\right]^{4}$ such that lines with a longer euclidean length were estimated to contain more dots $(M \mathrm{~s}=62.175$ vs. 57.43). Therefore, the results documented in Experiments $1-5$ were replicated with a different experimental procedure.

Moderating effect of clutter on the euclidean length effect. Consistent with our hypothesis that clutter would moderate the euclidean length effect, the interaction between euclidean length and clutter was significant $\left[F(1,118)=5.99, p<.05, S E_{\mathrm{ws}}=1.04\right]$. We expected that the euclidean length effect would be stronger when the configuration was less wide (i.e., had higher clutter). In support of this hypothesis, whereas euclidean length was significant under the high-clutter (low-width) configuration $[M s=57.76$ vs. $65.04 ; F(1,118)=18.57, p<$ $.001]$, it was only marginally significant under the lowclutter condition, in which the configuration was wider $[M s=57.11$ vs. $59.31 ; F(1,118)=2.06, p<.08]$.

Main effect of clutter. Consistent with previous research on distance perceptions, we also found that greater clutter led to higher numerosity estimates $[M \mathrm{~s}=61.4 \mathrm{vs}$. $\left.58.20 ; F(1,118)=8.55, p<.01, S E_{\mathrm{ws}}=1.1\right]$.

Moderating effect of other variables on the clutter effect. Follow-up tests for the euclidean length $x$ clutter interaction also showed that clutter significantly increased perceived numerosity under high euclidean length $[M \mathrm{~s}=$ 59.31 and $65.04 ; F(1,118)=10.57, p<.001]$ but not under low euclidean length $[M \mathrm{~s}=57.11$ and $57.76, p>$ $.25]$. This shows that clutter may have other moderating factors that need further research. Note that larger euclidean length (with constant clutter and total line length) also decreases euclidean width (see Figure 6A). Thus, euclidean width and/or euclidean length may moderate the clutter effect. This needs further research.

Effects of stimuli exposure time. Stimuli exposure time did not interact significantly with either euclidean length or clutter $(p>.25)$, showing that exposure time did not moderate the euclidean length or clutter effects or their interaction. However, exposure time had a direct effect on perceived numerosity: Perceived numerosity was higher under the longer exposure time $[M \mathrm{~s}=55.34$ and $64.27 ; F(1,118)=14.6, p<.001]$. This effect needs to be explored further in future research (see General Discussion). Cell means are given in Figure 6B.

\section{Discussion}

In Experiment 6 we were able to successfully replicate the euclidean length effect of Experiments 1-5 using short, controlled duration times and varying experimental procedure by showing subjects the stimuli one at a time. Thus, it appears that the euclidean length effect noted in Experiments $1-5$ is robust.

Further, in this experiment we proposed and demonstrated the moderating effect of euclidean width on the euclidean length effect in such a way that under conditions of low euclidean width (high clutter), the euclidean effect was stronger than under conditions of low euclidean width (low clutter). This result is consistent with our argument that the use of euclidean length as a source of information is inversely related to the salience of alternate sources of information that could be used to make a judgment.

However, as discussed in Experiment 1, clutter may exert a direct effect on numerosity estimates apart from the indirect effect through euclidean width proposed here. Therefore, we need additional evidence that euclidean width, rather than clutter per se, moderates the effect of euclidean length. The next experiment manipulated euclidean width by increasing total line length, holding clutter constant.

\section{EXPERIMENT 7 \\ The Interactive Effects of Total Line Length and Euclidean Length}

In this experiment, we varied euclidean width by manipulating total line length. We predicted that, given an equal number of line segments (clutter) and same euclidean length, the width of the configuration would be greater at longer line lengths (Figure 7A).

The only reason total line length and euclidean length differ is that we are investigating nonstraight lines. It can be argued that in much the same way that people use euclidean line length to estimate numerosity, they may use total line length to estimate numerosity. To isolate the effects of total line length and euclidean line length, we manipulated the two orthogonally. We were thus also able to study the direct effect of total line length on perceived numerosity.

To test the robustness of the euclidean length effect and the moderating effect of euclidean width on euclidean length, in this experiment we also manipulated a third factor-actual number of dots. We expected a direct effect of the number of dots on perceived numerosity: the greater the number of dots in an arrangement, the greater the estimate of numerosity. Further, on the basis of previous research on distance perceptions (Dainoff et al., 1974; McNamara et al., 1984; Newcombe \& Liben, 1982), we expected that the likelihood of underestimation of the number of dots would increase as the actual number of dots increases (or the likelihood of overestimation decreases), consistent with an overall regression to the mean effect. 


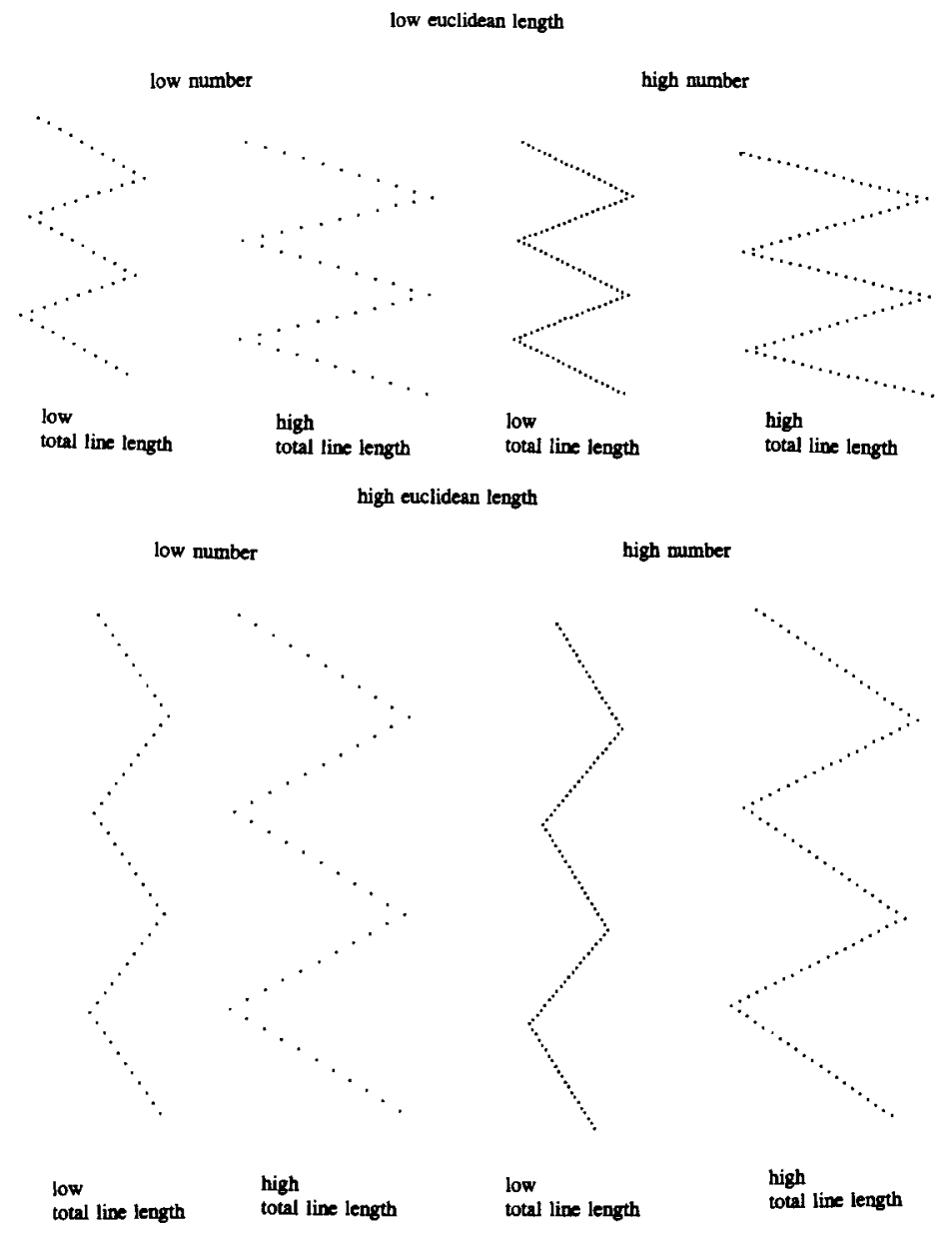

Figure 7A. Stimuli for Experiment 7: euclidean length, total line length, and actual number of dots.

\section{Method}

Subjects were 62 undergraduate students at a large business school who completed the experimental task during a regularly scheduled class for partial course credit. We used a $2 \times 2 \times 2$ (spatial stimuli: euclidean length, number of dots, and metric length) within-subjects design. Euclidean length was varied at 50 and $100 \mathrm{~mm}$, number of dots at 50 and 100 , and metric line length at 125 and $200 \mathrm{~mm}$ (Figure 7A). ${ }^{5}$ Given the results of Experiment 6 , which showed that the effect of euclidean length was smaller for this condition, clutter was kept constant at five segments so as to be more conservative. Hence, if we obtained a euclidean length effect at the lower clutter level, we would have greater confidence in our results. Further, to reduce any possibility of enumeration, time duration for stimuli viewing was reduced to $5 \mathrm{sec}$.

Note that manipulating the three variables also changed the angle between segments, the dot spacing, and the length of the line segments. For longer total line lengths or fewer number of dots (all else being the same), the spacing between dots increased. With longer euclidean length, the angle between segments increased. For longer total line length, the length of line segments increased.

The experiment procedure was the same as in Experiment 6 and took around $20 \mathrm{~min}$.

\section{Results}

Results from a $2 \times 2 \times 2$ repeated measures ANOVA are reported below. Effects hypothesized a priori are dis- cussed and are followed by a description of other significant effects. Cell means are graphically depicted in Figure 7B.

Euclidean length. As expected, lines with a shorter euclidean length were perceived to be shorter $(M=60.32)$ than lines with a longer euclidean length $[M=68.97$; $F(1,58)=27.78, p<.001, S E_{\mathrm{ws}}=2.32$; see Figure 7B] (See note 4.)

Number of dots. Not surprisingly, the main effect of actual number of dots was significant $[F(1,58)=123.71$, $p<.001, S E_{\mathrm{ws}}=3.9 \mathrm{]}$. Lines with 50 dots were perceived to have fewer dots than lines with 100 dots (overall $M \mathrm{~s}=$ 49.6 vs. 77.9). Further, consistent with the regression to the mean effect hypothesized, perceived numerosity of lines with 100 dots was underestimated (McNamara et al., 1984).

Total line length. The effect of total line length was significant: Longer lines were estimated to have more dots $(M=65.77)$ than shorter lines $[M=64.01 ; F(1,58)=$ $\left.2.90, p<.05, S E_{\mathrm{ws}}=1.87\right]$.

Interaction between total line length and euclidean length. On the basis of the results of Experiment 6, which showed that the effect of euclidean length diminished for 


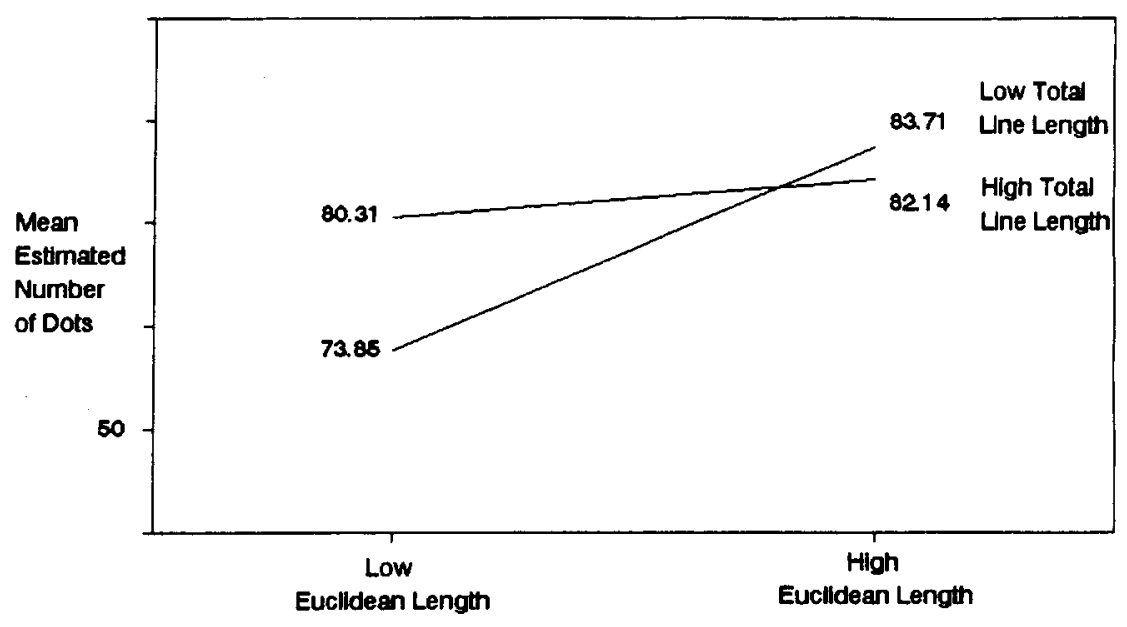

100 Actual Dots

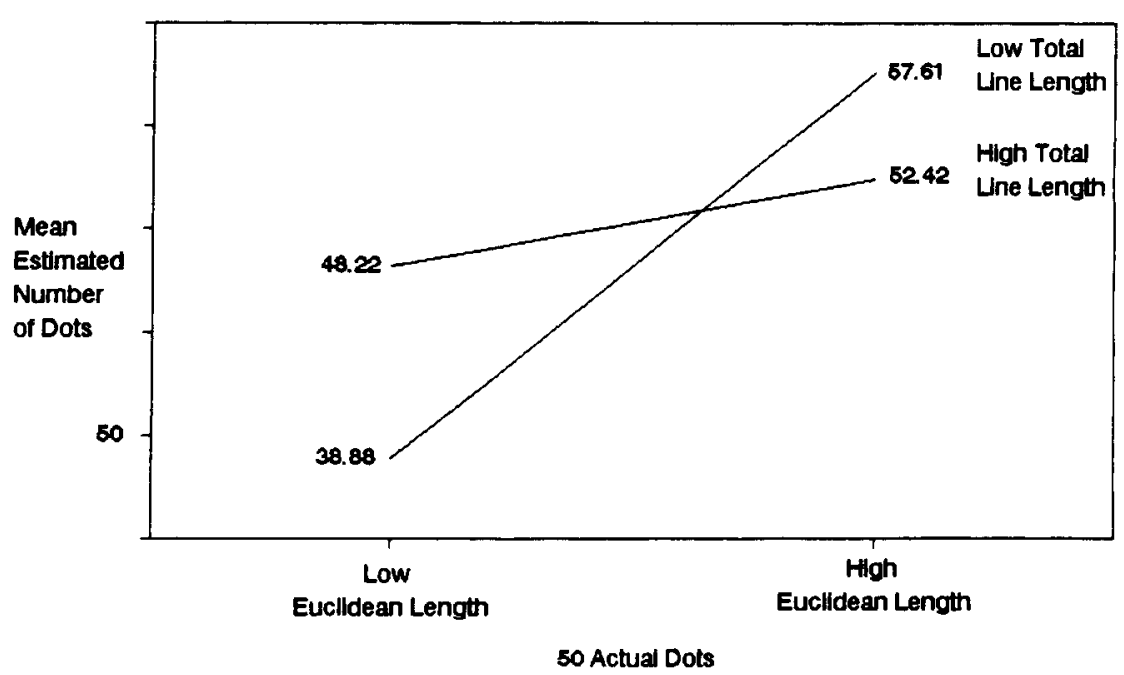

Figure 7B. Experiment 7: euclidean length, total line length, and actual number of dots.

wide configurations, we expected that the longer the total length of the configuration (i.e., the wider the configuration), the smaller the effect of the euclidean length. As expected, the interaction between these two factors was, indeed, significant $[F(1,58)=15.29, p<.001$, $\left.S E_{\mathrm{ws}}=2.04\right]$.

Follow-up analyses revealed that at short line lengths $(125 \mathrm{~mm})$, the main effect of euclidean length was significant: Lines with a shorter euclidean length were estimated to have fewer dots than were those with longer euclidean lengths $[M \mathrm{~s}=56.37$ vs. $70.66 ; F(1,60)=$ $40.66, p<.001]$. On the other hand, for long line lengths $(200 \mathrm{~mm})$, the overall effect of euclidean length-although in the expected direction did not approach conventional levels of significance $[M \mathrm{~s}=64.27$ vs. 67.28 ; $F(1,59)=1.66, p<.10]$. The weaker effect of euclidean length for lines with longer total length is consistent with the proposition that the wider the configuration, the lower the effect of euclidean length on numerosity perceptions.
Follow-up contrasts also revealed that higher total line length significantly increases perceived number under low euclidean length $(p<.001)$, but not under high euclidean length $(p=.12)$. Therefore, the effect of total line length on numerosity estimates does not appear to be robust.

Other results. The interaction between euclidean length and number of dots was significant $[F(1,58)=$ $\left.4.18, p<.05, S E_{\mathrm{ws}}=1.94\right]$. The form of this interaction showed that the euclidean length effect was stronger for configurations with 50 dots $[M \mathrm{~s}=43.55$ vs. 55.02 ; $F(1,61)=41.2, p<.001]$ than for configurations with 100 dots $[M \mathrm{~s}=77.08$ vs. $82.92 ; F(1,58)=5.65, p<$ $.025]$. This may be driven in part by the regression to the mean effect, whereby subjects underestimate large numbers. If the perceived number of dots is very large, subjects may downsize their perception overall for both the configuration with long euclidean lengths and the configuration with short euclidean lengths, thus diminishing the euclidean length effect. 
Relative effects. We were also interested in the relative size of effect for the different spatial features. Thus, we also analyzed the data using regression, with euclidean length, number of dots, line length, and all interactions of these variables as independent variables and estimate of number as the dependent variable. Note that this regression analysis was done across all subjects so that each of the 62 subjects yielded 8 data points. Therefore, the results of this regression analysis may differ slightly from the ANOVA results reported above.

The regression revealed that euclidean length (measured in millimeters) had the largest significant effect on perceived numerosity $(\beta=1.358, p<.025)$, actual number of dots the second largest $(\beta=1.29, p<.05)$, and line length the next largest effect, though it was only marginally significant $(\beta=0.465, p<.1)$. There was also a marginally significant negative interaction between euclidean length and total line length $(\beta=-0.0059, p<.1)$; the effect of euclidean length was weaker when total line length was higher (width was larger). This is consistent with our hypothesis that euclidean width moderates the euclidean length effect. Regression effects are reported in Table 1.

Experiments 6 and 7 show that reducing clutter and increasing line length, both of which increase euclidean width while holding the other constant, lead to diminished effects of euclidean length. Thus, euclidean width does indeed appear to be a moderator of the euclidean length effect. Further, it appears that euclidean length may be as good if not a better predictor of estimated numerosity than the actual number of dots.

\section{GENERAL DISCUSSION}

In this paper we wanted to explore factors that affect consumers' perceptions of numerosity of a line. Overall, our results suggest that euclidean length is a highly salient heuristic that subjects use to estirnate number in nonstraight lines. The robustness of the euclidean length effect was exhibited under different manipulations of euclidean length-namely, relative length of segments (Experiment 2), angle between segments (Experiment 3 ), and direction of segments (Experiment 4). We also found a significant effect under both memory-based judgments (Experiments 1-4) and stimuli-based judgments (Experiment 5), under uncontrolled stimuli exposure times (Experiments 1-5) and controlled and varied stimuli exposure times (Experiments 6 and 7), under exposure to multiple stimuli in a single trial (Experiments 1-5), and under exposure to single stimuli in multiple trials (Experiments 6 and 7).

However, the use of euclidean length as a heuristic in estimating number is inversely related to the salience of alternative heuristic inputs such as the euclidean width of the stimuli. Thus, when the width of the line configuration was varied by manipulating clutter (Experiment 6 ) or total line length (Experiment 7), the euclidean length effect was found to be stronger when euclidean width was low.
Table 1

\begin{tabular}{lccc}
\multicolumn{4}{c}{ Regression Results for Experiment 7 } \\
\hline & $\beta$ & $T$ & Sig $T$ \\
\hline Constant & -86.2135 & -1.846 & 0.0655 \\
Main Effects & & & \\
$\quad$ Euclidean length & 1.3579 & 2.298 & 0.0220 \\
$\quad \begin{array}{l}\text { Actual no. of dots } \\
\quad \text { Total line length }\end{array}$ & 1.2866 & 2.176 & 0.0301 \\
$\quad \begin{array}{l}\text { Interactions } \\
\quad \text { Euclidean length } \times\end{array}$ & & 1.660 & 0.0975 \\
$\quad$ total line length & -0.0059 & -1.661 & 0.0973 \\
$\quad \begin{array}{l}\text { Euclidean length } \times \\
\quad \text { actual no. of dots }\end{array}$ & -0.0093 & -1.246 & 0.2133 \\
$\quad$ Actual no. of dots $\times$ & & & \\
$\quad$ total line length & -0.0028 & -0.787 & 0.4315 \\
\hline
\end{tabular}

In Experiment 1, consistent with the clutter effect, we found that lines with intersections, gaps, or turns were perceived to have more dots than a control line without these features. In addition, consistent with the euclidean length effect, lines with intersections and gaps (lines with greater euclidean length) were estimated to have a greater number of dots than were lines with turns (lines with smaller euclidean length).

The euclidean length effect, controlling for clutter, was further explored in Experiments 2-4 by manipulating the effect of relative length of segments (Experiment 2), angle between segments (Experiment 3), and direction of segments (Experiment 4). We found consistent support for the euclidean length effect. In Experiment 5, we replicated these results in a context in which the stimuli were present in front of the subject, using a discrimination task rather than an estimation task.

Experiments 6 and 7 extended the spatial perception literature by proposing another spatial feature that may affect perception of number, namely the euclidean width of the line configuration. We proposed that increased width of the line increases its salience and decreases the use of euclidean length as a heuristic in estimating number. Thus, euclidean width moderates the euclidean length effect. Euclidean width of the line was varied through clutter (Experiment 6) and total line length (Experiment 7 ). Consistent with our hypothesis, the experiments showed that the higher the euclidean length/width ratio, the greater the effect of euclidean length.

In addition, Experiments 6 and 7 tested the direct effect of clutter and exposure time (Experiment 6), as well as total line length and number of dots (Experiment 7), on perceived numerosity. The results showed that increase in number of dots increases perceived numerosity, but is subject to a regression to the mean effect. Clutter was significant only for high euclidean length and not for low euclidean length, showing the need for further research on moderators of the clutter effect. In addition, we obtained mixed findings for the effect of total line length.

This paper extends the research on numerical perceptions by focusing on dotted lines, which have not received much attention in spite of their importance in decision making (see examples in introduction). We replicated 
the clutter effect and euclidean length effects found in the distance domain to the numerical domain and found results consistent with those in the distance domain. However, we demonstrated the moderating effect of euclidean width for the euclidean length effect. We also demonstrated the need for further research of the clutter effect to uncover potential moderating factors. Further, we found that the effect of total line length was not robust. Hence, our research reveals that the effects of euclidean length, euclidean width, total line length, clutter, angle between segments, dot spacing, and length of line segments have individual and interactive effects on perceived numerosity.

Researchers in this field might also further explore the degree to which higher exposure time leads to higher estimates of numerosity. Future research could also investigate whether the manner in which euclidean length (and width) affect estimates of dots follows a two-stage anchorand-adjust process. That is, do people anchor on euclidean length/width and then adjust their estimates based on the configuration of the line? An investigation of scan times in which subjects are instructed to scan each line of dots and to respond when they reach the end would elucidate this issue (cf. Kosslyn, Ball, \& Reiser, 1978). ${ }^{6}$

For the researcher studying biases in perceived length or number, our research suggests that experimental stimuli should control for the potential confounding effect of euclidean length between the end points of a line and for the euclidean width of the line. Thus, relative length of segments, angularity, and directionality of segments should be controlled for in investigations of how different spatial features lead to biased estimates of length and number, since they increase the euclidean length of the line. Also, clutter, total line length, and euclidean length should be controlled since they affect the width of the line.

At a general level, the research presented in this paper provides further evidence that people may use simplifying heuristics to make spatial judgments such as euclidean length and euclidean width. Reliance on these heuristics depends on the salience of alternative sources of information that could be used to make a judgment. Future research might investigate the manner in which simplifying 1-D heuristics, such as length and width, may be inappropriately combined to make 2-D judgments of area or 3-D judgments of volume.

\section{REFERENCES}

ALLEN, G. L. (1981). A developmental perspective on the effects of "subdividing" macrospatial experience. Journal of Experimental Psychology: Human Learning \& Memory, 7, 120-132.

ALLEN, G. L., \& KIRASIC, K. C. (1985). Effects of the cognitive organization of route knowledge on judgments of macrospatial distance. Memory \& Cognition, 13, 218-227.

ALLeN, G. L., SiEgEL, A. W., \& Rosinski, R. R. (1978). The role of perceptual context in structuring spatial knowledge. Journal of Experimental Psychology: Human Learning \& Memory, 4, 617-630.

ANTES, J. R., MCBRIDE, R. B., \& Collins, J. D. (1988). The effect of a new city traffic route on the cognitive maps of its residents. Environment \& Behavior, 20, 75-91.
AoKI, T. (1977). On the counting process of patterned dots. Tohoku Psychologica Folia, 36, 15-22.

atkinson, J., Cambell, F. W., \& Francis, M. R. (1976a). The dependence of the visual numerosity limit on orientation, color and grouping in the stimulus. Perception, 5, 335-344.

Atkinson, J., Cambell, F. W., \& Francis, M. R. (1976b). The magic number $4 \pm 0$ : A new look at visual numerosity judgments. Perception, 5, 327-334.

Beckwith, M., \& Restle, F. (1966). Process of enumeration. Psychological Review, 73, 437-444.

BLock, R. A. (1982). Temporal judgments and contextual change. Journal of Experimental Psychology: Learning, Memory, \& Cognition, $\mathbf{8}$, 530-544.

Brosvick, G. M., \& COHEN, B. D. (1988). The horizontal-vertical illusion and knowledge of the results. Perceptual \& Motor Skills, 67, 463-469.

BYRNE, R. W. (1979). Memory for urban geography. Quarterly Journal of Experimental Psychology, 31, 147-154.

Dainoff, M., Miskie, D., Wilson, C., \& Crane, P. (1974). Psychophysical measurement of environmental distance. In D. H. Carson (Ed.), Man-environment interactions: Evaluations and associations (EDRA 5 Proceedings). New York: Dowden, Hutchinson \& Ross

Dixon, P. (1978). Numerical comparison processes. Memory \& Cognition, 6, 454-461.

Finger, F. W., \& SPELt, D. K. (1947). The illustration of the horizontalvertical illusion. Journal of Experimental Psychology, 37, 243-250.

FreEMAN, F. N. (1912). Grouped objects as a concrete basis for the number idea. Elementary School Teacher, 12, 306-314.

Frith, C. D., \& Frith, U. (1972). The solitaire illusion: An illusion of numerosity. Perception \& Psychophysics, 11, 409-410.

GeLman, R. (1969). Conservation acquisition: A problem of learning to attend to relevant attributes. Journal of Experimental Child Psychology, 7, 167-187.

GiNSBURG, N. (1978). Perceived numerosity, item arrangement, and expectancy. American Journal of Psychology, 91, 267-273.

Herman, J. F., Norton, L. M., \& Klein, C. A. (1986). Children's distance estimates in a large-scale environment: A search for the route angularity effect. Environment \& Behavior, 18, 533-558.

KossLyn, S. M., BALL, T. M., \& ReIser, B. J. (1978). Psychology and living space. Journal of Experimental Psychology: Human Perception \& Performance, 4, 47-60.

Kosslyn, S. M., PICK, H. L., \& Fariello, G. R. (1974). Cognitive maps in children and men. Child Development, 45, 707-716.

LEE, T. R. (1973). Psychology and living space. In R. M. Downs \& D. Stea (Eds), Image and environment: Cognitive mapping and spatial behavior. Chicago: Aldine.

Loftus, G. R., \& MAsSON, M. E. J. (1994). Using confidence intervals in within-subject designs. Psychonomic Bulletin \& Review, 1, 476490

Luccio, R., \& Rodani, M. G. (1983). Illusioni di lunghezza e illusioni di numerosita [Illusions of length and illusions of numerosity]. $R i$ cerche di Psicologia, 7, 259-265.

Masin, S. C., \& Agostini, A. (1991). Handedness and space errors. Bulletin of the Psychonomic Society, 29, 301-303.

MCNAMARA, T. P. (1986). Mental representations of spatial relations. Cognitive Psychology, 18, 87-121

McNamara, T. P., Ratcliff, R., \& McKoon, G. (1984). The mental representation of knowledge acquired from maps. Journal of Experimental Psychology: Learning, Memory, \& Cognition, 10, 723-732.

MorRIs, R. B., \& RULE, S. J. (1988). Sequential judgement effects in magnitude estimation. Canadian Journal of Psychology, 42, 69-77.

NaSAR, J. L. (1985). Out of sight further from mind: Destination visibility and distance perceptions. Environment \& Behavior, 17, 627-639.

Newcombe, N., \& Liben, L. S. (1982). Barrier effects in the cognitive maps of children and adults. Journal of Experimental Child Psychology, 34, 46-58.

RaghubiR, P., \& Krishna, A. (1996, June). As the crow flies: Bias in consumers' map-based distance judgments. Journal of Consumer Research, 23, 26-39.

Sadalla, E. K., \& Magel, S. G. (1980). The perception of traversed distance. Environment \& Behavior, 12, 65-79. 
Sadalla, E. K., \& Staplin, L. J. (1980). The perception of traversed distance: Intersections. Environment \& Behavior, 12, 167-182.

Smitsman, A. W. (1982). Perception of number. International Journal of Behavioral Development, 5, 1-31.

THORNDYKE, P. W. (1981). Distance estimation from cognitive maps. Cognitive Psychology, 13, 526-550.

van Oeffelen, M. P., \& Vos, P. G. (1982). Configurational effects on the enumeration of dots: Counting by groups. Memory \& Cognition, 10, 396-404.

VAN OEFFELEN, M. P., \& Vos, P. G. (1984). Enumeration of dots: An eye movement analysis. Memory \& Cognition, 12, 607-612.

Wolters, G., van Kempen, H., \& WiJlhutzen, G.-J. (1987), Quantification of small number of dots: Subitizing or pattern recognition. American Journal of Psychology, 100, 225-237.

\section{NOTES}

1. This was surprising, because the Müller-Lyer illusion, which has documented that vertical lines are estimated to be longer than horizontal lines, has been found to be fairly robust for distance estimation tasks. It was only partially replicated to the numerosity domain.
2. Loftus and Masson (1994) have pointed out that the standard deviation around the mean may be misleading in analyses of data from within-subjects experiments. They suggested using the mean square of the interaction between subject and condition to determine the standard error for the confidence interval. In a repeated measures design, since there is just one factor (condition), the equivalent measure is the mean square within. Thus, although we report standard deviations around means, we also provide the within-subjects standard error, which is computed as sqrt $\left(\mathrm{MS}_{\mathrm{W}} / n\right)$, where $n$ is the number of subjects.

3. The subjects were actually exposed to four maps. However, we later found unequal dot spacing in one map and thus have not reported the results for this map.

4. Degrees of freedom for certain analyses are lower because of partial nonresponse by subjects.

5. Four of the stimuli in Experiment 7 were identical to those in Experiment 6 and are not repeated in Figure 7A

6. We thank Richard Block for this suggestion (see also Block, 1982).

(Manuscript received February 23, 1995; revision accepted for publication January 18, 1996.)

\section{Call for Papers on Teaching Demonstrations and Tutorials}

Many phenomena and concepts in behavioral research can be illustrated to students most effectively through software and video demonstrations and tutorials. A limiting factor in the use of such materials by instructors is knowledge of their existence and lack of easy accessibility. In part to remedy this situation, I am inviting submission of manuscripts describing demonstrations, tutorials, and experiments that can be used in instructional settings to further education regarding behavioral research. Manuscripts received by November 1, 1997, will be considered for a special issue or section of Behavior Research Methods, Instruments, \& Computers devoted to teaching demonstrations and tutorials. It is my hope that all individuals who have developed materials of this nature that would be of interest to a range of instructors will submit manuscripts and make the materials described in them readily available. The teaching demonstrations and tutorials of authors who consent will be placed at a Web site from which they can be accessed. Manuscripts should be sent in quadruplicate to Robert W. Proctor, Department of Psychology, Purdue University, 1364 Psychology Building, West Lafayette, IN 47907-1364.

Robert W. Proctor, Editor Behavior Research Methods, Instruments, \& Computers 